\title{
Robust linear semi-infinite programming duality under uncertainty ${ }^{\star}$
}

\section{Robust linear SIP duality}

\author{
Goberna M.A. ${ }^{1}$, Jeyakumar V. ${ }^{2}$, Li G. ${ }^{2}$, López M.A. ${ }^{1}$ \\ 1 Department of Statistics and Operations Research, University of Alicante, 03080 \\ Alicante, Spain \\ 2 Department of Applied Mathematics, University of New South Wales, Sydney \\ 2052, Australia
}

The date of receipt and acceptance will be inserted by the editor

\begin{abstract}
In this paper, we propose a duality theory for semi-infinite linear programming problems under uncertainty in the constraint functions, the objective function, or both, within the framework of robust optimization. We present robust duality by establishing strong duality between the robust counterpart of an uncertain semi-infinite linear program and the optimistic counterpart of its uncertain Lagrangian dual. We show that robust duality holds whenever a robust moment cone is closed and convex. We then show that the closed-convex robust moment cone condition in the case of constraint-wise uncertainty is in fact necessary and sufficient for robust duality in the sense that robust moment cone is closed and convex if and only if robust duality holds for every linear objective function of the program. In the case of uncertain problems with affinely parameterized data uncertainty, we establish that robust duality is easily satisfied under a Slater type constraint qualification. Consequently, we derive robust forms of the Farkas lemma for systems of uncertain semi-infinite linear inequalities.
\end{abstract}

Key words Robust optimization - semi-infinite linear programming - parameter uncertainty - robust duality - convex programming.

\footnotetext{
* This research was partially supported by ARC Discovery Project DP110102011 of Australia and by MICINN of Spain, Grant MTM2008-06695C03-0.
} 


\section{Introduction}

Duality theory has played a key role in the study of semi-infinite programming $[13,15,16,22]$ which traditionally assumes perfect information (that is, accurate values for the input quantities or system parameters), despite the reality that such precise knowledge is rarely available in practice for realworld optimization problems. The data of real-world optimization problems more often than not are uncertain (that is, they are not known exactly at the time of the decision) due to estimation errors, prediction errors or lack of information [3-6].

Robust optimization [2] provides a deterministic framework for studying mathematical programming problems under uncertainty. It is based on a description of uncertainty via sets, as opposed to probability distributions which are generally used in stochastic approaches [7,24]. A successful treatment of robust optimization approach for linear programming problems as well as convex optimization problems under data uncertainty has been given by Ben-Tal and Nemirovski [3-5], and El Ghaoui [12].

The present work was motivated by the recent development of robust duality theory $[1,21]$ for convex programming problems in the face of data uncertainty. To set the context of this work, consider a standard form of linear semi-infinite programming (SIP) problem in the absence of data uncertainty:

$$
\begin{array}{ll}
(S P) \text { inf } & \langle c, x\rangle \\
\text { s.t. } & \left\langle a_{t}, x\right\rangle \geq b_{t}, \forall t \in T,
\end{array}
$$

where $T$ is an arbitrary (possible infinite) index set, $c, a_{t} \in \mathbb{R}^{n}$, and $b_{t} \in$ $\mathbb{R}, t \in T$. The linear SIP problem in the face of data uncertainty in the constraints can be captured by the parameterized model problem

$$
\begin{aligned}
(U S P) & \inf \langle c, x\rangle \\
\text { s.t. } & \left\langle a_{t}\left(v_{t}\right), x\right\rangle \geq b_{t}\left(w_{t}\right), \forall t \in T,
\end{aligned}
$$

where $a_{t}: \mathcal{V}_{t} \rightarrow \mathbb{R}^{n}, b_{t}: \mathcal{W}_{t} \rightarrow \mathbb{R}, \mathcal{V}_{t} \subset \mathbb{R}^{q_{1}}, \mathcal{W}_{t} \subset \mathbb{R}^{q_{2}}, q_{1}, q_{2} \in \mathbb{N}$. The uncertain set-valued mapping $\mathcal{U}: T \rightrightarrows \mathbb{R}^{q}, q=q_{1}+q_{2}$, is defined as $\mathcal{U}_{t}:=\mathcal{V}_{t} \times \mathcal{W}_{t}$ for all $t \in T$. We represent by $u_{t}:=\left(v_{t}, w_{t}\right) \in \mathcal{V}_{t} \times$ $\mathcal{W}_{t}$ an arbitrary element of $\mathcal{U}_{t}$ or a variable ranging on $\mathcal{U}_{t}$. So, gph $\mathcal{U}=$ $\left\{\left(t, u_{t}\right): u_{t} \in \mathcal{U}_{t}, t \in T\right\}$. Throughout the paper, $u \in \mathcal{U}$ means that $u$ is a selection of $\mathcal{U}$, i.e., that $u: T \rightarrow \mathbb{R}^{q}$ and $u_{t} \in \mathcal{U}_{t}$ for all $t \in T$ ( $u$ can be also represented as $\left.\left(u_{t}\right)_{t \in T}\right)$. In stochastic programming $[7,24]$ each set $\mathcal{U}_{t}$ is equipped with a probability distribution and each selection of $\mathcal{U}$ determines a scenario for $(S P)$.

As an illustration of the model, consider the uncertain linear SIP problem:

$$
\inf _{\left(x_{1}, x_{2}\right) \in \mathbb{R}^{2}}\left\{x_{1}: a_{t}^{1} x_{1}+a_{t}^{2} x_{2} \geq b_{t}, t \in T\right\}
$$


where the data $a_{t}^{1}, a_{t}^{2}$ are uncertain, and for each $t \in T:=[0,1], a_{t}^{1} \in$ $[-1-2 t,-1+2 t], a_{t}^{2} \in[1 /(2+t), 1 /(2-t)]$ and $b_{t} \equiv-1$. Then, this uncertain problem can be captured by our model as

$$
\begin{array}{ll}
\text { inf } & x_{1} \\
\text { s.t. } & \left(-1+2 v_{t}^{1}\right) x_{1}+\left(\frac{1}{2+v_{t}^{2}}\right) x_{2} \geq-1, \forall t \in T,
\end{array}
$$

where $u_{t}=\left(v_{t}^{1}, v_{t}^{2}, w_{t}\right) \in \mathbb{R}^{3}$ is the uncertain parameter and $u_{t} \in \mathcal{U}_{t}:=$ $\mathcal{V}_{t} \times \mathcal{W}_{t}$ with $\mathcal{V}_{t}:=[-t, t] \times[-t, t]$ and $\mathcal{W}_{t}=\{0\}$. In this case, $a_{t}\left(v_{t}^{1}, v_{t}^{2}\right)=$ $\left(-1+2 v_{t}^{1}, 1 /\left(2+v_{t}^{2}\right)\right)$ and $b_{t}\left(w_{t}\right) \equiv-1$.

The robust counterpart of $(U S P)[1,3,5]$ is

$$
\begin{aligned}
(R S P) & \text { inf }\langle c, x\rangle \\
\text { s.t. } & \left\langle a_{t}\left(v_{t}\right), x\right\rangle \geq b_{t}\left(w_{t}\right), \forall\left(t, u_{t}\right) \in \operatorname{gph} \mathcal{U} .
\end{aligned}
$$

The problem (RSP) is indeed a linear SIP problem. We assume that (RSP) is a consistent problem with a closed and convex feasible set

$$
F:=\left\{x:\left\langle a_{t}\left(v_{t}\right), x\right\rangle \geq b_{t}\left(w_{t}\right), \forall\left(t, u_{t}\right) \in \operatorname{gph} \mathcal{U}\right\} \neq \emptyset .
$$

Now, for each fixed selection $u \in \mathcal{U}$, the Lagrangian dual of $(U S P)$ is

$$
(D P) \sup _{\lambda \in \mathbb{R}_{+}^{(T)}}\left\{\sum_{t \in T} \lambda_{t} b_{t}\left(w_{t}\right):-c+\sum_{t \in T} \lambda_{t} a_{t}\left(v_{t}\right)=0\right\},
$$

where $\mathbb{R}_{+}^{(T)}$ denotes the set of mappings $\lambda: T \rightarrow \mathbb{R}_{+}$(also denoted by $\left.\left(\lambda_{t}\right)_{t \in T}\right)$ such that $\lambda_{t}=0$ except for finitely many indexes, and $\sup \emptyset=-\infty$ by convention.

The optimistic counterpart [1,21] of the Lagrangian dual of $(D P)$ is given by

$$
(O D P) \sup _{\substack{u=\left(v_{t}, w_{t}\right)_{t \in T} \\ \lambda \in \mathbb{R}_{+}^{(T)}}}\left\{\sum_{t \in T} \lambda_{t} b_{t}\left(w_{t}\right):-c+\sum_{t \in T} \lambda_{t} a_{t}\left(v_{t}\right)=0\right\} .
$$

By construction,

$$
\inf (R S P) \geq \sup (O D P) .
$$

If $\inf (R S P)=-\infty$, then $(O D P)$ has no feasible solution, i.e.

$$
c \notin \bigcup_{u=\left(v_{t}, w_{t}\right)_{t \in T} \in \mathcal{U}} \operatorname{cocone}\left\{a_{t}\left(v_{t}\right), t \in T\right\},
$$

where co cone $\left\{a_{t}\left(v_{t}\right), t \in T\right\}$ denotes the convex cone generated by $\left\{a_{t}\left(v_{t}\right), t \in\right.$ $T\}$. We say that robust duality holds whenever $\inf (R S P)=\max (O D P)$, i.e.

$$
\inf \{\langle c, x\rangle: x \in F\}=\max _{\substack{u=\left(v_{t}, w_{t}\right)_{t \in T} \in \mathcal{U} \\ \lambda \in \mathbb{R}_{+}^{(T)}}}\left\{\sum_{t \in T} \lambda_{t} b_{t}\left(w_{t}\right):-c+\sum_{t \in T} \lambda_{t} a_{t}\left(v_{t}\right)=0\right\}
$$


whenever $\inf (R S P)$ is finite.

In this paper, we make the following key contributions: Firstly, we establish that robust duality holds for (USP) whenever the robust moment cone,

$$
M:=\bigcup_{u=\left(v_{t}, w_{t}\right)_{t \in T} \in \mathcal{U}} \operatorname{cocone}\left\{\left(a_{t}\left(v_{t}\right), b_{t}\left(w_{t}\right)\right), t \in T ;\left(0_{n},-1\right)\right\},
$$

is closed and convex. We further show that the closed-convex robust moment cone condition in the case of constraint uncertainty is in fact necessary and sufficient for robust duality in the sense that robust moment cone is closed and convex if and only if robust duality holds for every linear objective function of the program.

We also derive strong duality between the robust counterpart (RSP) and its standard (or Haar) dual problem [15] in terms of a robust characteristic cone, illustrating the link between the Haar dual and the optimistic dual.

Secondly, for the important case of affinely parametrized data uncertainty [2], we show that the convexity of the robust moment cone always holds and that it is closed under a robust Slater constraint qualification together with suitable topological requirements on the index set and the uncertainty set of the problem.

Thirdly, we derive robust forms of Farkas' lemma [10,11,17] for systems of uncertain semi-infinite linear inequalities in terms of the robust moment cone and the robust characteristic cones.

Finally, we extend our results to deal with uncertain linear SIP problems where data uncertainty occurs at both objective function and at constraints.

The organization of the paper is as follows. Section 2 provides robust duality theorems under geometric conditions in terms of robust moment and characteristic cones. Section 3 shows that these cone conditions are satisfied if and only if robust duality holds for every linear objective function. Section 4 presents robust Farkas' lemma for uncertain semi-infinite linear inequalities. Section 5 extends the results to deal with uncertain linear SIP problems where data uncertainty occurs at both objective function and constraints.

\section{Robust Duality}

Let us introduce the necessary notation. We denote by $\|\cdot\|$ and $\mathbb{B}_{n}$ the Euclidean norm and the open unit ball in $\mathbb{R}^{n}$. By $0_{n}$ we represent the null vector of $\mathbb{R}^{n}$. For a set $C \subset \mathbb{R}^{n}$, we define its convex hull co $C$ and conical hull cone $C$ as co $C=\left\{\sum_{i=1}^{m} \lambda_{i} c_{i}: \lambda_{i} \geq 0, \sum_{i=1}^{m} \lambda_{i}=1, c_{i} \in C, m \in \mathbb{N}\right\}$ and cone $C=\bigcup_{\lambda>0} \lambda C$, respectively. The topological closure of $C$ is cl $C$. Given $h: \mathbb{R}^{n} \rightarrow \mathbb{R} \cup\{+\infty\}$, such that $h \neq+\infty$, the epigraph of $h$ is

$$
\text { epi } h:=\left\{(x, r) \in \mathbb{R}^{n+1}: h(x) \leq r\right\}
$$


and the conjugate function of $h$ is $h^{*}: \mathbb{R}^{n} \rightarrow \mathbb{R} \cup\{+\infty\}$ such that

$$
h^{*}(v):=\sup \{\langle v, x\rangle-h(x) \mid x \in \operatorname{dom} h\} .
$$

The indicator and the support functions of $C$ are denoted respectively by $\delta_{C}$ and $\delta_{C}^{*}$.

Let $C \subset \mathbb{R}^{n}$ be a convex set and $h: C \rightarrow \mathbb{R}$. Identifying $h$ with its extension to $\mathbb{R}^{n}$ by defining $h(x):=+\infty$ for any $x \notin C, h$ is called convex when epi $h$ is convex, concave when $-h$ is convex, and affine when it is both convex and concave on $C$.

We define the robust moment cone of $(R S P)$ as

$$
M=\bigcup_{u=\left(v_{t}, w_{t}\right)_{t \in T} \in \mathcal{U}} \text { co cone }\left\{\left(a_{t}\left(v_{t}\right), b_{t}\left(w_{t}\right)\right), t \in T ;\left(0_{n},-1\right)\right\} .
$$

The next academic example shows that $M$ can be neither convex nor closed.

Example 1 Consider the simple uncertain linear SIP problem

$$
\begin{aligned}
& (S P) \text { inf }-x_{1}-x_{2} \\
& \text { s.t. }\left\langle a_{t}, x\right\rangle \geq b_{t}, \forall t \in T,
\end{aligned}
$$

where $T=[0,1], a_{0}$ is uncertain on the set

$$
\mathcal{V}_{0}=\{(\cos \alpha, \sin \alpha): \alpha \in[0,2 \pi] \cap \mathbb{Q}\}
$$

(a dense subset of the circle $\{x:\|x\|=1\}$ ) whereas the remaining data are deterministic: $b_{0}=-1$ and $\left(a_{t}, b_{t}\right)=\left(0_{2},-1\right)$ for $\left.\left.t \in\right] 0,1\right]$. This uncertain problem can be modeled as

$$
\begin{aligned}
& (U S P) \inf -x_{1}-x_{2} \\
& \text { s.t. }\left\langle a_{t}\left(v_{t}\right), x\right\rangle \geq b_{t}\left(w_{t}\right), \forall t \in T,
\end{aligned}
$$

with uncertain mapping $\mathcal{U}$ such that $\mathcal{U}_{0}=\mathcal{V}_{0} \times\{-1\}$ and $\mathcal{U}_{t}=\{(0,0,-1)\}$ for all $t \in] 0,1]$, and associated functions $a_{0}\left(v_{0}\right)=-v_{0}, a_{t}\left(v_{t}\right)=v_{t}=0_{2}$, for all $t \in] 0,1]$, and $b_{t}\left(w_{t}\right)=w_{t}=-1$, for all $t \in[0,1]$. Observe that there exists a one-to-one correspondence between the selections of $\mathcal{U}$ and the elements of $\mathcal{V}_{0}$ because the unique uncertain constraint is $\left\langle a_{0}, x\right\rangle \geq b_{0}$. So, the robust counterpart and the robust moment cone are

$$
\begin{aligned}
(R S P) \text { inf } & -x_{1}-x_{2} \\
\text { s.t. } & -\left\langle v_{0}, x\right\rangle \geq-1, \forall v_{0} \in \mathcal{V}_{0}, \\
& \left.\left.\left\langle 0_{2}, x\right\rangle \geq-1, \forall t \in\right] 0,1\right],
\end{aligned}
$$

and

$$
M=\bigcup_{v_{0} \in \mathcal{V}_{0}} \text { co cone }\left\{\left(-v_{0},-1\right),\left(0_{2},-1\right)\right\},
$$

respectively. Thus $M$ is the union of countable many 2-dimensional convex cones having a common edge on the vertical axis. Obviously, $M$ is neither convex nor closed. As $F=\mathrm{cl} \mathbb{B}$, the unique optimal solution is $(\sqrt{2} / 2, \sqrt{2} / 2)$ and $\min (R S P)=-\sqrt{2}$. Concerning the robust dual problem $(O D P)$, it is inconsistent because $(1,1) \notin$ co cone $\left\{v_{0}, 0_{2}\right\}=\mathbb{R}_{+}\left\{v_{0}\right\}$ whichever $v_{0} \in \mathcal{V}_{0}$ we consider $(\cos \alpha \neq \sin \alpha$ for any $\alpha \in[0,2 \pi] \cap \mathbb{Q})$, so that $\max (O D P)=-\infty$ by convention, i.e., there is an infinite duality gap. 
Theorem 1 If the robust moment cone $M$ is closed and convex, then

$$
\inf (R S P)=\max (O D P)
$$

Proof. Let $\alpha:=\inf (R S P) \in \mathbb{R}$. It follows that

$$
\left[\left\langle a_{t}\left(v_{t}\right), x\right\rangle \geq b_{t}\left(w_{t}\right), \forall\left(t, u_{t}\right) \in \operatorname{gph} \mathcal{U}\right] \Rightarrow\langle c, x\rangle-\alpha \geq 0,
$$

where $u_{t}=\left(v_{t}, w_{t}\right)$. Define $g: \mathbb{R}^{n} \rightarrow \mathbb{R}$ by

$$
g(x)=\sup _{\left(t, u_{t}\right) \in \operatorname{gph} \mathcal{U}}\left\{-\left\langle a_{t}\left(v_{t}\right), x\right\rangle+b_{t}\left(w_{t}\right)\right\}
$$

As $g$ is supremum of a collection of affine functions, $g$ is convex, $F=\{x$ : $g(x) \leq 0\}$, and (3) is equivalent to

$$
g(x) \leq 0 \Rightarrow\langle c, x\rangle-\alpha \geq 0 .
$$

Let $f(x):=\langle c, x\rangle+\delta_{F}(x)$. This implies that $f(x) \geq \alpha$ for all $x \in \mathbb{R}^{n}$. So, we have (see, e.g., [9] and [23])

$$
\begin{aligned}
(0,-\alpha) \in \operatorname{epi} f^{*} & =\operatorname{cl}\left(\operatorname{epi}(\langle c, .\rangle)^{*}+\operatorname{epi} \delta_{F}^{*}\right) \\
& =\operatorname{epi}(\langle c, .\rangle)^{*}+\operatorname{epi} \delta_{F}^{*} \\
& =\left(\{c\} \times \mathbb{R}_{+}\right)+\operatorname{epi} \delta_{F}^{*} .
\end{aligned}
$$

Note that $\delta_{[g \leq 0]}=\sup _{\lambda \geq 0}(\lambda g)$ and

$$
\operatorname{epi}\left(\sup _{i \in I} f_{i}\right)^{*}=\operatorname{clco}\left(\bigcup_{i \in I} \operatorname{epi} f_{i}^{*}\right)
$$

(see, e.g., $[14,(2.3)])$. It follows that

$$
\operatorname{epi} \delta_{F}^{*}=\operatorname{epi}\left(\sup _{\lambda \geq 0}(\lambda g)\right)^{*}=\operatorname{clco}\left(\bigcup_{\lambda \geq 0} \operatorname{epi}(\lambda g)^{*}\right) .
$$

As $\lambda g=\sup _{\left(t, u_{t}\right) \in \operatorname{gph} \mathcal{U}}\left\{\left\langle-\lambda a_{t}\left(v_{t}\right), x\right\rangle+\lambda b_{t}\left(w_{t}\right)\right\}$, we have by applying again (4)

$$
\operatorname{epi}(\lambda g)^{*}=\operatorname{clco}\left(\bigcup_{\left(t, u_{t}\right) \in \operatorname{gph} \mathcal{U}}\left[\left(-\lambda a_{t}\left(v_{t}\right),-\lambda b_{t}\left(w_{t}\right)\right)+\left\{0_{n}\right\} \times \mathbb{R}_{+}\right]\right) .
$$


This gives us that

$$
\begin{aligned}
\operatorname{epi} \delta_{F}^{*} & =\operatorname{clco}\left(\bigcup_{\lambda \geq 0} \operatorname{epi}(\lambda g)^{*}\right) \\
& =\operatorname{clco}\left(\bigcup_{\lambda \geq 0}\left\{\operatorname{clco}\left(\bigcup_{\left(t, u_{t}\right) \in \operatorname{gph} \mathcal{U}}\left[-\lambda\left(a_{t}\left(v_{t}\right), b_{t}\left(w_{t}\right)\right)+\left\{0_{n}\right\} \times \mathbb{R}_{+}\right]\right)\right\}\right) \\
& =\operatorname{clco}\left(\bigcup_{\lambda \geq 0} \bigcup_{\left(t, u_{t}\right) \in \operatorname{gph} \mathcal{U}}\left[-\lambda\left(a_{t}\left(v_{t}\right), b_{t}\left(w_{t}\right)\right)+\left\{0_{n}\right\} \times \mathbb{R}_{+}\right]\right) \\
& =-\operatorname{clco}\left(\bigcup_{\lambda \geq 0} \bigcup_{\left(t, u_{t}\right) \in \operatorname{gph} \mathcal{U}}\left[\lambda\left(a_{t}\left(v_{t}\right), b_{t}\left(w_{t}\right)\right)+\left\{0_{n}\right\} \times \mathbb{R}_{-}\right]\right) .
\end{aligned}
$$

On the other hand, Lemma 5.1 of Appendix shows that

$$
\operatorname{co}\left(\bigcup_{\lambda \geq 0} \bigcup_{\left(t, u_{t}\right) \in \operatorname{gph} \mathcal{U}}\left[\lambda\left(a_{t}\left(v_{t}\right), b_{t}\left(w_{t}\right)\right)+\left\{0_{n}\right\} \times \mathbb{R}_{-}\right]\right)=\operatorname{co} M,
$$

and hence

$$
\operatorname{epi} \delta_{F}^{*}=-\operatorname{clco} M
$$

As $M$ is closed and convex by our assumption, we have epi $\delta_{F}^{*}=-M$, and so,

$$
(0,-\alpha) \in\left(\{c\} \times \mathbb{R}_{+}\right)-M .
$$

This implies that there exists $\widehat{u}=\left(\widehat{v}_{t}, \widehat{w}_{t}\right)_{t \in T} \in \mathcal{U}$ and $\widehat{\lambda} \in \mathbb{R}_{+}^{(T)}$ such that

$$
c=\sum_{t \in T} \widehat{\lambda}_{t} a_{t}\left(\widehat{v}_{t}\right) \text { and }-\alpha \geq-\sum_{t \in T} \widehat{\lambda}_{t} b_{t}\left(\widehat{w}_{t}\right) .
$$

So, we see that $\alpha \leq \sum_{t \in T} \lambda_{t} b_{t}\left(w_{t}\right) \leq \max (O D P)$. Thus the conclusion follows from the weak duality, and we also conclude that $(\widehat{u}, \widehat{\lambda})$ is optimal for $(O D P)$.

Observe that $(O D P)$ is more easily manageable than the ordinary (or Haar) dual problem of $(R S P)$ :

$$
\begin{array}{cl}
(D R S P) \sup _{\lambda \in \mathbb{R}_{+}^{(\mathrm{gph} \mathcal{U})}} & \sum_{\left(t, u_{t}\right) \in \operatorname{gph} \mathcal{U}} \lambda_{\left(t, u_{t}\right)} b_{\left(t, u_{t}\right)}\left(w_{\left(t, u_{t}\right)}\right) \\
\text { s.t. } & -c+\sum_{\left(t, u_{t}\right) \in \operatorname{gph} \mathcal{U}} \lambda_{\left(t, u_{t}\right)} a_{\left(t, u_{t}\right)}\left(v_{\left(t, u_{t}\right)}\right)=0 .
\end{array}
$$

Now consider the so-called characteristic cone of $(R S P)$

$$
K=\operatorname{cocone}\left\{\left(a_{t}\left(v_{t}\right), b_{t}\left(w_{t}\right)\right),\left(t, u_{t}\right) \in \operatorname{gph} \mathcal{U} ;(0,-1)\right\} .
$$

Then $(D R S P)$ is equivalent to $\sup \{\gamma:(c, \gamma) \in K\}$ in the sense that both problems have the same optimal value and are simultaneously solvable or not. It is worth observing that $K=\operatorname{co} M$. In fact, $\operatorname{co} M \subset K$ because 
$M \subset K$ trivially and $K$ is convex. To show the reverse inclusion, take an arbitrary generator of $K$ different of $(0,-1) \in M$, say $\left(a_{s}\left(v_{s}\right), b_{s}\left(w_{s}\right)\right)$, with $\left(s, u_{s}\right) \in \operatorname{gph} \mathcal{U}$. As

$$
\left(a_{s}\left(v_{s}\right), b_{s}\left(w_{s}\right)\right) \in \operatorname{cocone}\left\{\left(a_{t}\left(v_{t}\right), b_{t}\left(w_{t}\right)\right), t \in T ;(0,-1)\right\},
$$

we have $\left(a_{s}\left(v_{s}\right), b_{s}\left(w_{s}\right)\right) \in M$. Thus, $K \subset \operatorname{co} M$ and so $K=\operatorname{co} M$.

From the linear SIP strong duality theorem, $\inf (R S P)=\max (D R S P)$ whenever $K$ is closed (see, e.g., [15, Chapter 8]). If $M$ is closed and convex, then $K=\operatorname{co} M=M$ is closed and so

$$
\begin{aligned}
\inf (R S P) & =\max (D R S P) \\
& =\max \{\gamma:(c, \gamma) \in K\} \\
& =\max \{\gamma:(c, \gamma) \in M\} \\
& =\max (O D P) .
\end{aligned}
$$

Thus, we have obtained an alternative proof of Theorem 1 appealing to linear SIP machinery.

In Example 1, the characteristic cone

$$
\begin{aligned}
K & =\text { co cone }\left\{\left(-v_{0},-1\right), v_{0} \in \mathcal{V}_{0} ;(0,-1)\right\} \\
& =\left\{x \in \mathbb{R}^{3}: x_{3}<-\sqrt{x_{1}^{2}+x_{2}^{2}}\right\} \cup \operatorname{cone}\left\{\mathcal{V}_{0} \times\{-1\}\right\}
\end{aligned}
$$

is not closed, $(D R S P)$ is not solvable and

$$
\inf (R S P)=-\sqrt{2}=\sup (D R S P)>\sup (O D P)=-\infty .
$$

$($ The equality $\inf (R S P)=\sup (D R S P)$ comes from [15, Theorem 8.1(v)].)

\section{Robust Moment Cones: Convexity and Closure}

We say that $(R S P)$ satisfies the convexity condition if for every $t \in T$, $a_{t}(\cdot)$ is affine, i.e., $a_{t}\left(v_{t}\right)=\left(a_{t}^{1}\left(v_{t}\right), \ldots, a_{t}^{n}\left(v_{t}\right)\right)$ and each $a_{t}^{j}(\cdot)$ is an affine function, $j=1, \ldots, n$, and $b_{t}(\cdot)$ is concave.

Proposition 1 Let $\mathcal{U}$ be convex-valued. Suppose that (RSP) satisfies the convexity condition. Then the robust moment cone $M$ is convex.

Proof. Let $a^{1}, a^{2} \in M$ and $\mu \in[0,1]$. Let $a:=\mu a^{1}+(1-\mu) a^{2}$ and denote $a=(z, \gamma) \in \mathbb{R}^{n} \times \mathbb{R}$, with $a^{1}=\left(z^{1}, \gamma_{1}\right) \in \mathbb{R}^{n} \times \mathbb{R}$ and $a^{2}=\left(z^{2}, \gamma_{2}\right) \in \mathbb{R}^{n} \times \mathbb{R}$. Then, there exist $u^{1}=\left(v_{t}^{1}, w_{t}^{1}\right)_{t \in T} \in \mathcal{U}, \lambda^{1}=\left(\lambda_{t}^{1}\right)_{t \in T} \in \mathbb{R}_{+}^{(T)}$, and $\alpha_{1} \leq 0$ such that

$$
\left(z^{1}, \gamma_{1}\right)=\sum_{t \in T} \lambda_{t}^{1}\left(a_{t}\left(v_{t}^{1}\right), b_{t}\left(w_{t}^{1}\right)\right)+\left(0, \alpha_{1}\right) .
$$

Similarly, there exist $u^{2}=\left(v_{t}^{2}, w_{t}^{2}\right)_{t \in T} \in \mathcal{U}, \lambda^{2}=\left(\lambda_{t}^{2}\right)_{t \in T} \in \mathbb{R}_{+}^{(T)}$ and $\alpha_{2} \leq 0$ such that

$$
\left(z^{2}, \gamma_{2}\right)=\sum_{t \in T} \lambda_{t}^{2}\left(a_{t}\left(v_{t}^{2}\right), b_{t}\left(w_{t}^{2}\right)\right)+\left(0, \alpha_{2}\right) .
$$


Then, we have

$$
\mu z^{1}+(1-\mu) z^{2}=\sum_{t \in T}\left(\mu \lambda_{t}^{1} a_{t}\left(v_{t}^{1}\right)+(1-\mu) \lambda_{t}^{2} a_{t}\left(v_{t}^{2}\right)\right)
$$

and

$$
\mu \gamma_{1}+(1-\mu) \gamma_{2}=\sum_{t \in T}\left(\mu \lambda_{t}^{1} b_{t}\left(w_{t}^{1}\right)+(1-\mu) \lambda_{t}^{2} b_{t}\left(w_{t}^{2}\right)\right)+\mu \alpha_{1}+(1-\mu) \alpha_{2} .
$$

We associate with $t \in T$ the scalar $\lambda_{t}:=\mu \lambda_{t}^{1}+(1-\mu) \lambda_{t}^{2}$ and the vector

$$
\left(v_{t}, w_{t}\right):= \begin{cases}\left(v_{t}^{1}, w_{t}^{1}\right), & \text { if } \lambda_{t}=0 \\ \frac{\mu \lambda_{t}^{1}}{\lambda_{t}}\left(v_{t}^{1}, w_{t}^{1}\right)+\frac{(1-\mu) \lambda_{t}^{2}}{\lambda_{t}}\left(v_{t}^{2}, w_{t}^{2}\right), & \text { if } \lambda_{t}>0\end{cases}
$$

Then $\left(v_{t}, w_{t}\right) \in \mathcal{U}_{t}$ and

$$
\mu \lambda_{t}^{1}\left(v_{t}^{1}, w_{t}^{1}\right)+(1-\mu) \lambda_{t}^{2}\left(v_{t}^{2}, w_{t}^{2}\right)=\lambda_{t}\left(v_{t}, w_{t}\right) .
$$

By our convexity assumption, we see that, for each $t \in T$,

$$
\mu \lambda_{t}^{1} a_{t}\left(v_{t}^{1}\right)+(1-\mu) \lambda_{t}^{2} a_{t}\left(v_{t}^{2}\right)=\lambda_{t} a_{t}\left(v_{t}\right),
$$

and

$$
\mu \lambda_{t}^{1} b_{t}\left(w_{t}^{1}\right)+(1-\mu) \lambda_{t}^{2} b_{t}\left(w_{t}^{2}\right) \leq \lambda_{t} b_{t}\left(w_{t}\right) .
$$

Then, there will exist $\rho \leq 0$ such that

$$
\begin{aligned}
a & =(z, \gamma)=\mu\left(z^{1}, \gamma_{1}\right)+(1-\mu)\left(z^{2}, \gamma_{2}\right) \\
& =\left(\mu z^{1}+(1-\mu) z^{2}, \mu \gamma_{1}+(1-\mu) \gamma_{2}\right) \\
& =\left(\sum_{t \in T} \lambda_{t} a_{t}\left(v_{t}\right), \sum_{t \in T} \lambda_{t} b_{t}\left(w_{t}\right)\right)+\left(0_{n}, \mu \alpha_{1}+(1-\mu) \alpha_{2}+\rho\right) \\
& =\sum_{t \in T} \lambda_{t}\left(a_{t}\left(v_{t}\right), b_{t}\left(w_{t}\right)\right)+\left(-\mu \alpha_{1}-(1-\mu) \alpha_{2}-\rho\right)\left(0_{n},-1\right),
\end{aligned}
$$

and this implies that $a=(z, \gamma) \in M$.

In particular, the robust moment cone $M$ is convex in the important affinely data parametrization case [2], i.e.

$$
\mathcal{U}_{t}=\left\{\left(a_{t}, b_{t}\right)=\left(a_{t}^{0}, b_{t}^{0}\right)+\sum_{j=1}^{q} u_{t}^{j}\left(a_{t}^{j}, b_{t}^{j}\right):\left(u_{t}^{1}, \ldots, u_{t}^{q}\right) \in Z_{t}\right\},
$$

where $Z_{t}$ is closed and convex for each $t \in T$. In this case, $\mathcal{U}$ is convex-valued and the convexity condition holds with $b_{t}(\cdot)$ being also affine.

It is easy to see from Example 1 that $M$ may not be convex when $\mathcal{U}$ is not convex-valued. In fact, in this example, $\mathcal{U}_{0}=\mathcal{V}_{0} \times\{0\}$ is not even connected. 
The set-valued mapping $\mathcal{U}: T \rightrightarrows \mathbb{R}^{q}$, with $(T, d)$ being a metric space, is said to be (Hausdorff) upper semicontinuous at $t \in T$ if for any $\epsilon>0$ there exists $\eta>0$ such that

$$
\mathcal{U}_{s} \subset \mathcal{U}_{t}+\epsilon \mathbb{B}_{q} \forall s \in T \text { with } d(s, t) \leq \eta .
$$

In particular, $\mathcal{U}$ is uniformly upper semicontinuous on $T$ if for any $\epsilon>0$ there exists $\eta>0$ such that

$$
\mathcal{U}_{s} \subset \mathcal{U}_{t}+\epsilon \mathbb{B}_{q} \forall s, t \in T \text { with } d(s, t) \leq \eta .
$$

If, additionally, $T$ is compact, then there exists a finite set $\left\{t_{1}, \ldots, t_{m}\right\} \subset T$ such that $d\left(t,\left\{t_{1}, \ldots, t_{m}\right\}\right)<\eta$ for all $t \in T$. Then, $\mathcal{U}_{s} \subset \bigcup_{i=1, \ldots, m}\left(\mathcal{U}_{t_{i}}+\epsilon \mathbb{B}_{q}\right)$ $\forall s \in T$, so that gph $\mathcal{U}$ is bounded whenever $\mathcal{U}$ is compact-valued, i.e., for each $t \in T, \mathcal{U}_{t}$ is a compact set.

We say that $(R S P)$ satisfies the Slater condition when there exists $x_{0} \in$ $\mathbb{R}^{n}$ (called Slater point) such that $\left\langle a_{t}\left(v_{t}\right), x_{0}\right\rangle>b_{t}\left(w_{t}\right)$ for all $\left(t, u_{t}\right) \in \operatorname{gph} \mathcal{U}$, i.e. when there exists a strict solution of the constraint system of $(R S P)$.

Proposition 2 Suppose that the following three assumptions hold:

(i) $T$ is a compact metric space;

(ii) $\mathcal{U}$ is compact-valued and uniformly upper semicontinuous on $T$;

(iii) the mapping

$$
\operatorname{gph} \mathcal{U} \ni\left(t, u_{t}\right) \mapsto\left(a_{t}\left(v_{t}\right), b_{t}\left(w_{t}\right)\right) \in \mathbb{R}^{n+1}
$$

is continuous on gph $\mathcal{U}$;

(iv) $(R S P)$ satisfies the Slater condition.

Then, the robust moment cone $M$ is closed.

Remark (before the proof) (ii) and (iii) conjointly imply that $a_{t}\left(\mathcal{V}_{t}\right)$ and $b_{t}\left(\mathcal{W}_{t}\right)$ are compact. From now on we shall call (iii) continuity condition.

Proof. Let

$$
\left(z^{k}, r_{k}\right) \in M=\bigcup_{u \in \mathcal{U}} \operatorname{cocone}\left\{\left(a_{t}\left(v_{t}\right), b_{t}\left(w_{t}\right)\right) \cup\left(0_{n},-1\right): t \in T\right\}, k=1,2, \ldots,
$$

such that $\left(z^{k}, r_{k}\right) \rightarrow(z, r)$. Then, for each $k$, there exists $u^{k} \in \mathcal{U}$, with $u_{t}^{k}=\left(v_{t}^{k}, w_{t}^{k}\right) \in \mathcal{U}_{t}$ for all $t \in T$, such that

$$
\left(z^{k}, r_{k}\right) \in \operatorname{cocone}\left\{\left(a_{t}\left(v_{t}^{k}\right), b_{t}\left(w_{t}^{k}\right)\right) \cup\left(0_{n},-1\right): t \in T\right\} .
$$

From the Carathéodory theorem, we can find $\lambda_{i}^{k} \geq 0, i=1, \ldots, n+2$, $\mu_{k} \geq 0,\left\{t_{1}^{k}, \ldots, t_{n+2}^{k}\right\} \subset T$ and $\left(v_{i}^{k}, w_{i}^{k}\right) \in \mathcal{U}_{t_{i}^{k}}, i=1, \ldots, n+2$, such that

$$
\left(z^{k}, r_{k}\right)=\sum_{i=1}^{n+2} \lambda_{i}^{k}\left(a_{t_{i}^{k}}\left(v_{i}^{k}\right), b_{t_{i}^{k}}\left(w_{i}^{k}\right)\right)+\mu_{k}\left(0_{n},-1\right) .
$$

As $T$ is compact, we may assume that $t_{i}^{k} \rightarrow t_{i} \in T, i=1, \ldots, n+2$. 
Fix $i=1, \ldots, n+2$. Since we are assuming that $\mathcal{U}$ is uniformly upper semicontinuous and so, for any $\epsilon>0$, there exists $\eta>0$ such that

$$
\mathcal{U}_{t} \subseteq \mathcal{U}_{t_{i}}+\epsilon \mathbb{B}_{q}, \text { for all } t \text { such that } d\left(t, t_{i}\right) \leq \eta
$$

It follows that

$$
d\left(\left(v_{i}^{k}, w_{i}^{k}\right), \mathcal{U}_{t_{i}}\right) \rightarrow 0 \text { as } k \rightarrow \infty .
$$

Since $\mathcal{U}_{t_{i}}$ is compact, we may assume the existence of $\left(v_{i}, w_{i}\right) \in \mathcal{U}_{t_{i}}$ such that

$$
\left(v_{i}^{k}, w_{i}^{k}\right) \rightarrow\left(v_{i}, w_{i}\right) \text { as } k \rightarrow \infty .
$$

As $(R S P)$ satisfies (iii)

$$
\left(a_{t_{i}^{k}}\left(v_{i}^{k}\right), b_{t_{i}^{k}}\left(w_{i}^{k}\right)\right) \rightarrow\left(a_{t_{i}}\left(v_{i}\right), b_{t_{i}}\left(w_{i}\right)\right) \text { as } k \rightarrow \infty
$$

Now, we show that $l_{k}:=\sum_{i=1}^{n+2} \lambda_{i}^{k}+\mu^{k}$ is bounded. Granting this, by passing to subsequence if necessary, we may assume that

$$
\lambda_{i}^{k} \rightarrow \lambda_{i} \in \mathbb{R}_{+} \text {and } \mu_{k} \rightarrow \mu \in \mathbb{R}_{+}
$$

as each $\lambda_{i}^{k}$ and $\mu_{k}$ are non-negative. Then, passing to the limit in (12) we have

$$
(z, r)=\sum_{i=1}^{n+2} \lambda_{i}\left(a_{t_{i}}\left(v_{i}\right), b_{t_{i}}\left(w_{i}\right)\right)+\mu\left(0_{n},-1\right) \in M
$$

Therefore, the robust moment cone $M$ is closed.

To show the boundedness of $l_{k}$, we proceed by the method of contradiction and assume without loss of generality that $l_{k}:=\sum_{i=1}^{n+2} \lambda_{i}^{k}+\mu^{k} \rightarrow+\infty$. By passing to subsequence if necessary, we may assume that $\frac{\lambda_{i}^{k}}{l_{k}} \rightarrow \bar{\lambda}_{i} \in \mathbb{R}_{+}$, $\frac{\mu^{k}}{l_{k}} \rightarrow \bar{\mu} \in \mathbb{R}_{+}$and

$$
\sum_{i=1}^{n+2} \bar{\lambda}_{i}+\bar{\mu}=1
$$

Dividing by $l_{k}$ both members of (12) and passing to the limit, we obtain that

$$
\left(0_{n}, 0\right)=\sum_{i=1}^{n+2} \bar{\lambda}_{i}\left(a_{t_{i}}\left(v_{i}\right), b_{t_{i}}\left(w_{i}\right)\right)+\bar{\mu}\left(0_{n},-1\right) .
$$

So, we have $\sum_{i=1}^{n+2} \bar{\lambda}_{i} a_{t_{i}}\left(v_{i}\right)=0_{n}$ and $\sum_{i=1}^{n+2} \bar{\lambda}_{i} b_{t_{i}}\left(w_{i}\right)=\bar{\mu}$ and so, taking a Slater point $x_{0}$, we have

$$
\sum_{i=1}^{n+2} \bar{\lambda}_{i}\left(\left\langle a_{t_{i}}\left(v_{i}\right), x_{0}\right\rangle-b_{t_{i}}\left(w_{i}\right)\right)=-\bar{\mu} \leq 0 .
$$


On the other hand, since $\left(v_{i}, w_{i}\right) \in \mathcal{U}_{t_{i}}$, assumption (iii) implies that $\left\langle a_{t_{i}}\left(v_{i}\right), x_{0}\right\rangle-$ $b_{t_{i}}\left(w_{i}\right)>0$ for all $i=1, \ldots, n+2$. Note that $\left(\bar{\lambda}_{1}, \ldots, \bar{\lambda}_{n+2}\right) \neq 0_{n+2}$ (otherwise, $\bar{\mu}=\sum_{i=1}^{n+2} \bar{\lambda}_{i} r_{i}=0$ and so, $\left(\bar{\lambda}_{1}, \ldots, \bar{\lambda}_{n+2}, \bar{\mu}\right)=0_{n+3}$ which contradicts (14)). This implies that

$$
\sum_{i=1}^{n+2} \bar{\lambda}_{i}\left(\left\langle a_{t_{i}}\left(v_{i}\right), x_{0}\right\rangle-b_{t_{i}}\left(w_{i}\right)\right)>0 .
$$

This is a contradiction and so, $\left\{l_{k}\right\}$ is a bounded sequence.

Example 1 violates assumptions (ii) and (iii) in Proposition 2 because $\mathcal{U}_{0}$ is neither compact nor convex, $\mathcal{U}$ is not upper semicontinuous at 0 , and the function in (11) is not continuous. In fact, if we consider the sequence $\left(t_{k}, u_{t_{k}}\right)=\left(1 / k,\left(0_{2},-1\right)\right)$ which converges to $\left(0,\left(0_{2},-1\right)\right)$, we have $\left(a_{t_{k}}\left(v_{t_{k}}\right), b_{t_{k}}\left(w_{t_{k}}\right)\right)=\left(0_{2},-1\right)$ which does not converge to $\left(a_{0}\left(v_{0}\right), b_{0}\left(w_{0}\right)\right)$ as $v_{0}=0_{2} \notin \mathcal{V}_{0}$.

As an immediate consequence of the previous results, we obtain the following sufficient condition for robust duality. In the special case when $|T|<+\infty$, this result collapses to the robust strong duality result for linear programming problems in [1].

Corollary 1 Suppose that the following assumptions hold:

(i) $T$ is a compact metric space;

(ii) $\mathcal{U}$ is compact-convex-valued and uniformly upper semicontinuous on $T$;

(iii) (RSP) satisfies the convexity, the continuity and the Slater conditions.

Then, the robust duality holds, i.e. $\inf (R S P)=\max (O D P)$.

Proof. The conclusion follows from Theorem 1, Proposition 1, and Proposition 2.

We now present an example verifying Corollary 1.

Example 2 Let $T=[0,1]$ and consider the following uncertain linear SIP problem:

$$
\begin{array}{ll}
\text { inf } & x \\
\text { s.t. } & a_{t} x \geq b_{t}, t \in T,
\end{array}
$$

where the data $a_{t}, b_{t}$ are uncertain, $a_{t} \in[-1-2 t,-1+2 t]$ and $b_{t} \equiv-1$. This uncertain problem can be captured by our model as

$$
\begin{array}{ll}
\text { inf } & x \\
\text { s.t. } & a_{t}\left(v_{t}\right) x \geq b_{t}\left(w_{t}\right), t \in T,
\end{array}
$$

where $\left(v_{t}, w_{t}\right)$ are the uncertain parameter, $a_{t}\left(v_{t}\right):=-1+2 v_{t}, v_{t} \in \mathcal{V}_{t}:=$ $[-t, t]$ and $b_{t}\left(w_{t}\right):=-1$. Let $\mathcal{U}_{t}:=\mathcal{V}_{t} \times\{0\}$. Then the robust counterpart is

$$
\begin{array}{ll}
\inf & x \\
\text { s.t. } & a_{t}\left(v_{t}\right) x \geq b_{t}\left(w_{t}\right), \forall\left(t, u_{t}\right) \in \operatorname{gph} \mathcal{U} .
\end{array}
$$


It can be verified that the feasible set is $[-1,1 / 3]$. So, the optimal value of the robust counterpart is -1 . The optimistic counterpart of the dual problem is

$$
(O D P) \quad \sup _{\lambda \in \mathbb{R}_{+}^{(T)}, u \in \mathcal{U}}\left\{-\sum_{t \in T} \lambda_{t}:-1+\sum_{t \in T} \lambda_{t}\left(-1+2 v_{t}\right)=0\right\} .
$$

Let $\bar{\lambda} \in \mathbb{R}_{+}^{(T)}$ such that $\bar{\lambda}_{1}=1$ and $\bar{\lambda}_{t}=0$ for all $t \in T \backslash\{1\}$. Let $u=(v, 0) \in$ $\mathcal{U}$ such that $v_{1}=1$. Then $-1+\sum_{t \in T} \bar{\lambda}_{t}\left(-1+2 v_{t}\right)=-1+\bar{\lambda}_{1}\left(-1+2 v_{1}\right)=0$ and $-\sum_{t \in T} \bar{\lambda}_{t}=-1$. So, $\max (O D P)=-1$ and the robust strong duality holds. In fact, $M=$ co cone $\{(-3,-1),(-1,-1)\}$ is closed and convex. Finally, one can see that all the conditions in the preceding corollary are satisfied.

The following theorem shows that our assumption is indeed a characterization for robust strong duality in the sense that "the convexity and closedness of the robust moment cone" hold if and only if the robust strong duality holds for each linear objective function of $(R S P)$.

Theorem 2 The following statements are equivalent to each other:

(i) For all $c \in \mathbb{R}^{n}$,

$$
\inf \{\langle c, x\rangle: x \in F\}=\max _{\lambda \in \mathbb{R}_{+}^{(T)}, u \in \mathcal{U}}\left\{\sum_{t \in T} \lambda_{t} b_{t}\left(w_{t}\right):-c+\sum_{t \in T} \lambda_{t} a_{t}\left(v_{t}\right)=0\right\} .
$$

(ii) The robust moment cone $M$ is closed and convex.

Proof. [(ii) $\Rightarrow$ (i)] It follows by Theorem 1 .

$[(\mathrm{i}) \Rightarrow$ (ii)] We proceed by contradiction and let

$$
\left(c_{0}, r_{0}\right) \in(\operatorname{clco} M) \backslash M \text {. }
$$

So, (6) implies that $\left(-c_{0},-r_{0}\right) \in \operatorname{epi} \delta_{F}^{*}$ where $F:=\{x: g(x) \leq 0\}$ and

$$
g(x)=\sup _{\left(t, u_{t}\right) \in \operatorname{gph} \mathcal{U}}\left\{-\left\langle a_{t}\left(v_{t}\right), x\right\rangle+b_{t}\left(w_{t}\right)\right\} .
$$

Thus, we have

$$
\delta_{F}^{*}\left(-c_{0}\right) \leq-r_{0} .
$$

So, for every $x \in F$,

$$
\left\langle c_{0}, x\right\rangle \geq r_{0}
$$

It follows that

$$
r_{0} \leq \inf \left\{\left\langle c_{0}, x\right\rangle:\left\langle a_{t}\left(v_{t}\right), x\right\rangle \geq b_{t}\left(w_{t}\right), \forall\left(t, u_{t}\right) \in \operatorname{gph} \mathcal{U}\right\} .
$$

Thus, the statement (i) gives that

$$
r_{0} \leq \max _{\lambda \in \mathbb{R}_{+}^{(T)}, u \in \mathcal{U}}\left\{\sum_{t \in T} \lambda_{t} b_{t}\left(w_{t}\right):-c_{0}+\sum_{t \in T} \lambda_{t} a_{t}\left(v_{t}\right)=0\right\} .
$$


and so, there exists $\bar{\lambda} \in \mathbb{R}_{+}^{(T)}$ and $\left(\bar{v}_{t}, \bar{w}_{t}\right) \in \mathcal{U}_{t}$ for all $t \in T$, with $-c_{0}+$ $\sum_{t \in T} \bar{\lambda}_{t} a_{t}\left(\bar{v}_{t}\right)=0$ and such that

$$
r_{0} \leq \sum_{t \in T} \bar{\lambda}_{t} b_{t}\left(\bar{w}_{t}\right)
$$

This shows that

$$
\left.\left(z_{0}, r_{0}\right) \in \operatorname{cocone}\left\{\left(a_{t}\left(\bar{v}_{t}\right), b_{t}\left(\bar{w}_{t}\right)\right), t \in T ;(0,-1)\right\} \subset M\right\},
$$

which constitutes a contradiction.

\section{Robust Semi-infinite Farkas' Lemma}

In this Section, as consequences of robust duality results of previous Sections, we derive two forms of robust Farkas' lemma for a system of uncertain semi-infinite linear inequalities. Related results may be found in $[8,18-21]$.

Corollary 2 (Robust Farkas' Lemma: Characterization I) The following statements are equivalent to each other:

(i) For all $c \in \mathbb{R}^{n}$, the following statements are equivalent:

1) $\left[\left\langle a_{t}\left(v_{t}\right), x\right\rangle \geq b_{t}\left(w_{t}\right), \forall\left(t, u_{t}\right) \in \operatorname{gph} \mathcal{U}\right] \Rightarrow\langle c, x\rangle \geq r$

2) $\exists \lambda=\left(\lambda_{t}\right)_{t \in T} \in \mathbb{R}_{+}^{(T)}$ and $\left(v_{t}, w_{t}\right) \in \mathcal{U}_{t}, t \in T$,

$$
\text { such that }\left\{\begin{array}{l}
-c+\sum_{t \in T} \lambda_{t} a_{t}\left(v_{t}\right)=0 \\
\text { and } \sum_{t \in T} \lambda_{t} b_{t}\left(w_{t}\right) \geq r
\end{array}\right.
$$

(ii) The robust moment cone $M$ is closed and convex.

Proof. The conclusion follows immediately from Theorem 2.

Next we compare the previous results with similar ones involving the characteristic cone $K$ defined in (8) and the standard dual (DRSP) introduced in (7) instead of $M$ and $(O D P)$, respectively.

Recall that, the assumptions in Proposition 1 and 2 guarantee that the robust moment cone $M$ is convex and closed (and so, the characteristic cone $K$ is also closed). In the following, we show that the assumptions in Proposition 2 alone ensure that the characteristic cone $K$ is closed.

Proposition 3 Under the same assumptions as in Proposition 2, the characteristic cone $K$ is closed and $\inf (R S P)=\max (D R S P)$

Proof. We first prove that the characteristic cone $K$ of the robust linear SIP problem is closed. Condition (ii) in Proposition 2 guarantees the closedness of the index set gph $\mathcal{U}$. In fact, let $\left\{\left(t_{r}, v_{r}, w_{r}\right)\right\} \subset \operatorname{gph} \mathcal{U}$ be a sequence such that $\left(t_{r}, v_{r}, w_{r}\right) \rightarrow(t, v, w) \in T \times \mathbb{R}^{q_{1}+q_{2}}$. Then $\left(v_{r}, w_{r}\right) \in \mathcal{U}_{t_{r}}$ for all $r \in \mathbb{N}, t_{r} \rightarrow t$, and $\left(v_{r}, w_{r}\right) \rightarrow(v, w)$. Assume that $(t, v, w) \notin \operatorname{gph} \mathcal{U}$, i.e. that $(v, w) \notin \mathcal{U}_{t}$. Since $\mathcal{U}_{t}$ is closed, there exists $\epsilon>0$ such that $(v, w) \notin \mathcal{U}_{t}+\epsilon \mathbb{B}_{q}$. 
Let $\eta>0$ be such that $\mathcal{U}_{s} \subset \mathcal{U}_{t}+\frac{\epsilon}{2} \mathbb{B}_{q}$ for any $s \in T$ with $d(s, t) \leq \eta$. We have $d\left(t_{r}, t\right) \leq \eta$ and $d\left(\left(v_{r}, w_{r}\right),(v, w)\right)<\frac{\epsilon}{2}$ for sufficiently large $r$, so that $\left(v_{r}, w_{r}\right) \in \mathcal{U}_{t_{r}} \subset \mathcal{U}_{t}+\frac{\epsilon}{2} \mathbb{B}_{q}$ and $(v, w) \in \mathcal{U}_{t}+\epsilon \mathbb{B}_{q}$ (contradiction). We have also seen that condition (ii) implies the boundedness of gph $\mathcal{U}$, which turns out to be compact. On the other hand, condition (iii) states that the mapping from gph $\mathcal{U}$ to $\mathbb{R}^{n+1}$ such that $\left(t, u_{t}\right) \mapsto\left(a_{t}\left(v_{t}\right), b_{t}\left(w_{t}\right)\right)$ is continuous and so, the set

$$
\left\{\left(a_{t}\left(v_{t}\right), b_{t}\left(w_{t}\right)\right),\left(t, u_{t}\right) \in \operatorname{gph} \mathcal{U}\right\}
$$

is compact. As we are assuming that the Slater condition holds, $K$ is closed by [15, Theorem 5.3 (ii)]. Finally, the closedness of $K$ and [15, (8.5)-(8.6)] imply $\inf (R S P)=\max (D R S P)$.

We have seen that, under the assumptions of Proposition 2, the characteristic cone $K$ is closed, and this entails that any Slater point is an interior point of $F$ ([15, Theorem 5.9 (iv)]). Thus, $F$ is full dimensional.

Proposition 4 The following statements are equivalent to each other:

(i) For all $c \in \mathbb{R}^{n}$,

$$
\begin{aligned}
\inf \{\langle c, x\rangle: x \in F\}= & \max _{\lambda \in \mathbb{R}_{+}^{(\operatorname{gph} \mathcal{U})}}\left\{\sum_{\left(t, u_{t}\right) \in \operatorname{gph} \mathcal{U}} \lambda_{\left(t, u_{t}\right)} b_{\left(t, u_{t}\right)}\left(w_{\left(t, u_{t}\right)}\right):\right. \\
& \left.-c+\sum_{\left(t, u_{t}\right) \in \operatorname{gph} \mathcal{U}} \lambda_{\left(t, u_{t}\right)} a_{\left(t, u_{t}\right)}\left(v_{\left(t, u_{t}\right)}\right)=0\right\} .
\end{aligned}
$$

(ii) The characteristic cone $K$ is closed.

Proof. It is straightforward consequence of [15, Theorem 8.4].

Corollary 3 (Robust Farkas' Lemma: Characterization II) The following statements are equivalent to each other:

(i) For all $c \in \mathbb{R}^{n}$, the following statements are equivalent:

1) $\left[\left\langle a_{t}\left(v_{t}\right), x\right\rangle \geq b_{t}\left(w_{t}\right), \forall\left(t, u_{t}\right) \in \operatorname{gph} \mathcal{U}\right] \Rightarrow\langle c, x\rangle \geq r$.

$\left.2^{\prime}\right) \exists \lambda=\left(\lambda_{t}\right)_{t \in T} \in \mathbb{R}_{+}^{(\mathrm{gph} \mathcal{U})}$

$$
\text { such that }\left\{\begin{array}{c}
-c+\sum_{\left(t, u_{t}\right) \in \operatorname{gph} \mathcal{U}} \lambda_{\left(t, u_{t}\right)} a_{\left(t, u_{t}\right)}\left(v_{\left(t, u_{t}\right)}\right)=0, \\
\text { and } \sum_{\left(t, u_{t}\right) \in \operatorname{gph} \mathcal{U}} \lambda_{\left(t, u_{t}\right)} b_{\left(t, u_{t}\right)}\left(w_{\left(t, u_{t}\right)}\right) \geq r .
\end{array}\right.
$$

(ii) The characteristic cone $K$ is closed.

Proof. It is straightforward consequence of Proposition 4.

\section{Uncertainty in all the data}

In this section, we consider linear SIP problems where uncertainty occurs in both objective function and in the constraints. This situation can be modeled as the parameterized linear SIP problem

$$
\begin{array}{cc}
(\widetilde{U S P}) \inf _{x \in \mathbb{R}^{n}} & \left\langle c\left(v_{s}\right), x\right\rangle \\
\text { s.t. } & \left\langle a_{t}\left(v_{t}\right), x\right\rangle \geq b_{t}\left(w_{t}\right), \forall t \in T,
\end{array}
$$


where the uncertain parameter $v_{s}$ ranges on some set $Z \subset \mathbb{R}^{q_{3}}$ and $c: Z \rightarrow$ $\mathbb{R}^{n}$. For convenience, we always assume that $s \notin T$. The problem $(\widetilde{U S P})$ can be equivalently rewritten as

$$
\begin{array}{ll}
\inf _{(x, y) \in \mathbb{R}^{n} \times \mathbb{R}} & y \\
\text { s.t. } & \left\langle a_{t}\left(v_{t}\right), x\right\rangle \geq b_{t}\left(w_{t}\right), \forall t \in T, \\
& y-\left\langle c\left(v_{s}\right), x\right\rangle \geq 0
\end{array}
$$

So, the robust (or pessimistic) counterpart of $(\widetilde{U S P})$ can be formulated as

$$
\begin{array}{cl}
(\widetilde{R S P}) \inf _{(x, y) \in \mathbb{R}^{n} \times \mathbb{R}} & y \\
\text { s.t. } & \left\langle a_{t}\left(v_{t}\right), x\right\rangle \geq b_{t}\left(w_{t}\right), \forall\left(t, u_{t}\right) \in \operatorname{gph} \mathcal{U}, \\
& y-\left\langle c\left(v_{s}\right), x\right\rangle \geq 0, \forall v_{s} \in Z,
\end{array}
$$

whose decision space is $\mathbb{R} \times \mathbb{R}^{n}$. In other words, the robust counterpart $(\widetilde{R S P})$ is indeed a linear SIP problem with $n+1$ decision variables $y, x_{1}, \ldots, x_{n}$ and deterministic objective function as follows:

$$
\begin{array}{cl}
(\widetilde{R S P}) \inf _{(x, y) \in \mathbb{R}^{n} \times \mathbb{R}} & \left\langle\left(1,0_{n}\right),(y, x)\right\rangle \\
\text { s.t. } & \left\langle\widetilde{a}_{t}\left(v_{t}\right),(y, x)\right\rangle \geq \widetilde{b}_{t}\left(w_{t}\right), \forall\left(t, u_{t}\right) \in \operatorname{gph} \widetilde{\mathcal{U}},
\end{array}
$$

where $x=\left(x_{1}, \ldots, x_{n}\right), \widetilde{T}:=T \cup\{s\}($ as $s \notin T), q:=q_{1}+q_{2}+q_{3}, \widetilde{\mathcal{U}}: \widetilde{T} \rightrightarrows \mathbb{R}^{q}$ is the extension of $\mathcal{U}: T \rightrightarrows \mathbb{R}^{q}$ to $\widetilde{T}$ which results of defining $\widetilde{\mathcal{U}}_{s}:=Z \times\{1\}$, and the functions $\widetilde{a}_{t}: \mathcal{V}_{t} \rightarrow \mathbb{R}^{n+1}$ and $\widetilde{b}_{t}: \mathcal{W}_{t} \rightarrow \mathbb{R}$ are

$$
\widetilde{a}_{t}\left(v_{t}\right):= \begin{cases}\left(1,-c\left(v_{s}\right)\right), & \text { if } t=s, \\ \left(0, a_{t}\left(v_{t}\right)\right), & \text { otherwise }\end{cases}
$$

and

$$
\widetilde{b}_{t}\left(w_{t}\right):=\left\{\begin{array}{l}
0, \quad \text { if } t=s, \\
b_{t}\left(w_{t}\right), \text { otherwise. }
\end{array}\right.
$$

The constraint system of the optimistic counterpart for $(\widetilde{R S P})$, say $(\widetilde{O D P})$, is

$$
\sum_{t \in T} \lambda_{t}\left(\begin{array}{c}
0 \\
a_{t}\left(v_{t}\right)
\end{array}\right)+\mu\left(\begin{array}{c}
1 \\
-c\left(v_{s}\right)
\end{array}\right)=\left(\begin{array}{l}
1 \\
0
\end{array}\right),
$$

where $v_{t} \in \mathcal{V}_{t}, t \in T, v_{s} \in Z, \lambda \in \mathbb{R}_{+}^{(T)}$, and $\mu \in \mathbb{R}_{+}$. Eliminating $\mu=1$ we get

$$
(\widetilde{O D P}) \sup _{u \in \mathcal{U}, v_{s} \in Z, \lambda \in \mathbb{R}_{+}^{(T)}}\left\{\sum_{t \in T} \lambda_{t} b_{t}\left(w_{t}\right):-c\left(v_{s}\right)+\sum_{t \in T} \lambda_{t} a_{t}\left(v_{t}\right)=0\right\} .
$$

Finally, the robust moment cone of $(\widetilde{R S P})$ is

$$
\begin{aligned}
\widetilde{M} & =\bigcup_{u \in \widetilde{\mathcal{U}}} \operatorname{cocone}\left\{\left(\widetilde{a}_{t}\left(v_{t}\right), \widetilde{b}_{t}\left(w_{t}\right)\right), t \in \widetilde{T} ;(0,-1)\right\} \\
& =\bigcup_{\substack{u=\left(v_{t}, w_{t}\right)_{t \in T} \in \mathcal{U} \\
v_{s} \in Z}} \operatorname{cocone}\left\{\left(0, a_{t}\left(v_{t}\right), b_{t}\left(w_{t}\right)\right), t \in T ;\left(1,-c\left(v_{s}\right), 0\right),(0,0,-1)\right\} .
\end{aligned}
$$


Proposition 5 Suppose that $\mathcal{U}$ is convex-valued, that $Z$ is a convex set, and that all the components of $c(\cdot)$ and of $a_{t}(\cdot), t \in T$, are affine whereas the functions $b_{t}(\cdot), t \in T$, are concave. Then the robust moment cone $\widetilde{M}$ is convex.

Proof. By assumption, $\widetilde{\mathcal{U}}_{t}=\mathcal{U}_{t}$ is convex for all $t \in T$ and $\widetilde{\mathcal{U}}_{s}=Z \times\{1\}$ is a convex too, so that $\widetilde{\mathcal{U}}$ is convex-valued. Moreover, $(\widetilde{R S P})$ satisfies the convexity condition. The conclusion follows from Proposition 1.

It easily follows from Proposition 5 that the robust moment cone $\widetilde{M}$ is convex in the case where the data $\left(a_{t}, b_{t}, c\right)$ are affine.

Proposition 6 Suppose that the following assumptions hold:

(i) $T$ is a compact metric space;

(ii) $\mathcal{U}$ is compact-valued and uniformly upper semicontinuous on $T$, and $Z$ is compact;

(iii) $c: Z \rightarrow \mathbb{R}^{n}$ is continuous and the mapping from gph $\mathcal{U}$ in $\mathbb{R}^{n+1}$ such that $\left(t, u_{t}\right) \mapsto\left(a_{t}\left(v_{t}\right), b_{t}\left(w_{t}\right)\right) \in \mathbb{R}^{n+1}$ is continuous on $\operatorname{gph} \mathcal{U}$; $\operatorname{gph} \mathcal{U}$.

(iv) there exists $x_{0} \in \mathbb{R}^{n}$ such that $\left\langle a_{t}\left(v_{t}\right), x_{0}\right\rangle>b_{t}\left(w_{t}\right)$ for all $\left(t, u_{t}\right) \in$

Then, the robust moment cone $\widetilde{M}$ is closed.

Proof. By assumption (i), we can take a scalar $\delta$ greater than the diameter of $T$. We extend the distance $d$ to $\widetilde{T}$ by defining $d(s, t)=d(t, s)=\delta$ for all $t \in T$ and $d(s, s)=0$. Obviously, $(\widetilde{T}, d)$ is a compact metric space.

By assumption (ii), $\widetilde{\mathcal{U}}$ is compact-valued since $\mathcal{U}$ is compact-valued and $\widetilde{\mathcal{U}}_{s}=Z \times\{1\}$. Given $\epsilon>0$, there exists $\eta>0$ such that $\mathcal{U}_{t^{\prime}} \subset \mathcal{U}_{t}+\epsilon \mathbb{B}_{q}$ for all $t^{\prime}, t \in T$ with $d\left(t^{\prime}, t\right) \leq \eta<\delta$. So, if $t^{\prime}, t \in \widetilde{T}$ are such that $t^{\prime} \neq s \neq t$, we have $\widetilde{\mathcal{U}}_{t^{\prime}} \subset \widetilde{\mathcal{U}}_{t}+\epsilon \mathbb{B}_{q}$. Otherwise, $d\left(t^{\prime}, t\right)<\delta$ implies that $t^{\prime}=t=s$, so that $\widetilde{\mathcal{U}}_{t^{\prime}} \subset \widetilde{\mathcal{U}}_{t}+\epsilon \mathbb{B}_{q}$ trivially holds. So, $\widetilde{\mathcal{U}}$ is uniformly upper semicontinuous on $\widetilde{T}$.

By (iii), and by the isolation of $s$ in $\widetilde{T}$, the mapping

$$
\operatorname{gph} \widetilde{\mathcal{U}} \ni\left(t, u_{t}\right) \mapsto\left(\widetilde{a}_{t}\left(v_{t}\right), \widetilde{b}_{t}\left(w_{t}\right)\right) \in \mathbb{R}^{n+2}
$$

is continuous on gph $\widetilde{\mathcal{U}}$. Moreover, $\left(y_{0}, x_{0}\right)$ is a Slater point for $(\widetilde{R S P})$ whenever $y_{0}>\max _{v_{s} \in Z}\left\langle c\left(v_{s}\right), x_{0}\right\rangle$.

The conclusion follows from Proposition 2 applied to $(\widetilde{R S P})$ and $\widetilde{M}$.

Theorem 3 Suppose that the following assumptions hold:

(i) $T$ is a compact metric space;

(ii) $\mathcal{U}$ is compact-convex-valued and uniformly upper semicontinuous on $T$, and $Z$ is a compact and convex subset of $\mathbb{R}^{q_{3}}$;

(iii) $c: Z \rightarrow \mathbb{R}^{n}$ is continuous and the mapping from gph $\mathcal{U}$ in $\mathbb{R}^{n+1}$ such that $\left(t, u_{t}\right) \mapsto\left(a_{t}\left(v_{t}\right), b_{t}\left(w_{t}\right)\right) \in \mathbb{R}^{n+1}$ is continuous;

(iv) The components of $c(\cdot)$ and of $a_{t}(\cdot), t \in T$, are affine whereas the functions $b_{t}(\cdot), t \in T$, are concave; 
(v) There exists $x_{0} \in \mathbb{R}^{n}$ such that $\left\langle a_{t}\left(v_{t}\right), x_{0}\right\rangle>b_{t}\left(w_{t}\right)$ for all $\left(t, u_{t}\right) \in$ gph $\mathcal{U}$.

Then, the robust duality holds, i.e. $\inf (\widetilde{R S P})=\max (\widetilde{O D P})$.

Proof From Proposition 5 and Proposition 6, we see that the robust moment cone $\tilde{M}$ is closed convex. Thus the conclusion follows from Theorem 1 .

\section{Uncertainty in the objective function}

In the particular case of uncertainty in the objective and deterministic constraints, the parameterized associated problem is

$$
\begin{aligned}
(\widehat{U S P}) \inf & \left\langle c\left(v_{s}\right), x\right\rangle \\
\text { s.t. } & \left\langle a_{t}, x\right\rangle \geq b_{t}, \forall t \in T,
\end{aligned}
$$

where $c: Z \rightarrow \mathbb{R}^{n}, Z \subset \mathbb{R}^{q_{3}}$, and $a_{t} \in \mathbb{R}^{n}, b_{t} \in \mathbb{R}$ for all $t \in T, s \notin T$. Its robust counterpart is the particular case of $(\widetilde{R S P})$ in $(18)$ in which

$$
\widetilde{\mathcal{U}}_{t}=\left\{\begin{array}{l}
Z \times\{1\}, \text { if } t=s, \\
\{t\} \times\{t\}, \text { otherwise, }
\end{array}\right.
$$

(so that $\mathcal{V}_{t}=\{t\}$ and $\mathcal{W}_{t}=\{t\}$ ),

$$
\begin{gathered}
\widetilde{a}_{t}\left(v_{t}\right):= \begin{cases}\left(1,-c\left(v_{s}\right)\right), & \text { if } t=s, \\
\left(0, a_{t}\right), & \text { otherwise, }\end{cases} \\
\widetilde{b}_{t}\left(w_{t}\right):= \begin{cases}0, & \text { if } t=s, \\
b_{t}, & \text { otherwise, }\end{cases}
\end{gathered}
$$

the robust moment cone is

$$
\widetilde{M}=\bigcup_{v_{s} \in Z} \text { co cone }\left\{\left(0, a_{t}, b_{t}\right), t \in T ;\left(1,-c\left(v_{s}\right), 0\right),(0,0,-1)\right\},
$$

and finally, the optimistic counterpart is

$$
(\widetilde{O D P}) \sup _{v_{s} \in Z, \lambda \in \mathbb{R}_{+}^{(T)}}\left\{\sum_{t \in T} \lambda_{t} b_{t}:-c\left(v_{s}\right)+\sum_{t \in T} \lambda_{t} a_{t}=0\right\} .
$$
$(\widehat{U S P})$.

As a consequence of Proposition 5 and 6 , we obtain robust duality for

Corollary 4 Suppose that the following assumptions hold:

(i) $T$ is a compact metric space and the functions $t \mapsto a_{t}$ and $t \mapsto b_{t}$ are continuous on $T$;

(ii) $Z$ is compact and convex, and $c: Z \rightarrow \mathbb{R}^{n}$ is continuous;

(iii) all the components of $c(\cdot)$ are affine;

(iv) there exists $x_{0} \in \mathbb{R}^{n}$ such that $\left\langle a_{t}, x_{0}\right\rangle>b_{t}$ for all $t \in T$.

Then, the robust duality holds, i.e. $\inf (\widetilde{R S P})=\max (\widetilde{O D P})$.

Proof The conclusion follows from Proposition 4.1, Proposition 4.2 and Theorem 3. 


\section{Appendix}

In this Section, we provide a technical lemma which is used in the proof of Theorem 1. This lemma provides a characterization for the convex hull of the robust moment cone.

Lemma 1 Let $T$ be an arbitrary index set, $a_{t}: \mathcal{V}_{t} \rightarrow \mathbb{R}^{n}, b_{t}: \mathcal{W}_{t} \rightarrow \mathbb{R}$, $\mathcal{V}_{t} \subset \mathbb{R}^{q_{1}}, \mathcal{W}_{t} \subset \mathbb{R}^{q_{2}}, t \in T$ and $q_{1}, q_{2} \in \mathbb{N}$. Let $u_{t}=\left(v_{t}, w_{t}\right), t \in T$, and let the robust moment cone $M$ be defined as in (2). Then, we have

$$
\begin{aligned}
& \operatorname{co} M=\operatorname{co}\left(\bigcup_{\lambda \geq 0} \bigcup_{\left(t, u_{t}\right) \in \operatorname{gph} \mathcal{U}}\left(\left(\lambda a_{t}\left(v_{t}\right), \lambda b_{t}\left(w_{t}\right)\right)+\{0\} \times(-\infty, 0]\right)\right) . \\
& \text { Proof. []] Let }(z, r) \in \bigcup_{\lambda \geq 0} \bigcup_{\left(t, u_{t}\right) \in \operatorname{gph} \mathcal{U}}\left(\left(\lambda a_{t}\left(v_{t}\right), \lambda b_{t}\left(w_{t}\right)\right)+\{0\} \times(-\infty, 0]\right) .
\end{aligned}
$$

Then, there exists $\lambda \geq 0, s \in T$ and $\left(v_{s}, w_{s}\right) \in \mathcal{U}_{s}$ such that

$$
(z, r) \in\left(\lambda a_{s}\left(v_{s}\right), \lambda b_{s}\left(w_{s}\right)\right)+\{0\} \times(-\infty, 0] .
$$

So,

$$
z=\lambda a_{s}\left(v_{s}\right) \text { and } r \leq \lambda b_{s}\left(w_{s}\right)
$$

Letting $\mu=\lambda b_{s}\left(w_{s}\right)-r \geq 0$, this implies that

$$
\begin{aligned}
(z, r) & =\left(\left(\lambda a_{s}\left(v_{s}\right), \lambda b_{s}\left(w_{s}\right)\right)+\mu(0,-1)\right. \\
& \in \bigcup_{u=\left(v_{t}, w_{t}\right)_{t \in T} \in \mathcal{U}} \operatorname{cocone}\left\{\left(a_{t}\left(v_{t}\right), b_{t}\left(w_{t}\right)\right), t \in T ;(0,-1)\right\} .
\end{aligned}
$$

Thus, the definition of $M$ gives us that

$$
\bigcup_{\lambda \geq 0} \bigcup_{\left(t, u_{t}\right) \in \operatorname{gph} \mathcal{U}}\left(\left(\lambda a_{t}\left(v_{t}\right), \lambda b_{t}\left(w_{t}\right)\right)+\{0\} \times(-\infty, 0]\right) \subset M,
$$

and so,

$$
\operatorname{co}\left(\bigcup_{\lambda \geq 0} \bigcup_{\left(t, u_{t}\right) \in \operatorname{gph} \mathcal{U}}\left(\left(\lambda a_{t}\left(v_{t}\right), \lambda b_{t}\left(w_{t}\right)\right)+\{0\} \times(-\infty, 0]\right)\right) \subseteq \operatorname{co} M
$$

$[\subseteq]$ Let $(z, r) \in M$. Then, there exists $u=\left(v_{t}, w_{t}\right)_{t \in T} \in \operatorname{gph} \mathcal{U}$ such that

$$
(z, r) \in \text { co cone }\left\{\left(a_{t}\left(v_{t}\right), b_{t}\left(w_{t}\right)\right), t \in T ;(0,-1)\right\} .
$$

Note that

$$
\begin{aligned}
\operatorname{cone}\left\{\left(a_{t}\left(v_{t}\right), b_{t}\left(w_{t}\right)\right) \cup(0,-1)\right. & : t \in T\} \\
& \subseteq \bigcup_{\lambda \geq 0} \bigcup_{\left(t, u_{t}\right) \in \operatorname{gph} \mathcal{U}}\left(\left(\lambda a_{t}\left(v_{t}\right), \lambda b_{t}\left(w_{t}\right)\right)+\{0\} \times(-\infty, 0]\right) .
\end{aligned}
$$


This gives us that

$$
(z, r) \in \operatorname{co}\left(\bigcup_{\lambda \geq 0} \bigcup_{\left(t, u_{t}\right) \in \operatorname{gph} \mathcal{U}}\left(\left(\lambda a_{t}\left(v_{t}\right), \lambda b_{t}\left(w_{t}\right)\right)+\{0\} \times(-\infty, 0]\right)\right) .
$$

Thus, we have

$$
M \subset \operatorname{co}\left(\bigcup_{\lambda \geq 0} \bigcup_{\left(t, u_{t}\right) \in \operatorname{gph} \mathcal{U}}\left(\left(\lambda a_{t}\left(v_{t}\right), \lambda b_{t}\left(w_{t}\right)\right)+\{0\} \times(-\infty, 0]\right)\right) .
$$

and so,

$$
\operatorname{co} M \subset \operatorname{co}\left(\bigcup_{\lambda \geq 0} \bigcup_{\left(t, u_{t}\right) \in \operatorname{gph} \mathcal{U}}\left(\left(\lambda a_{t}\left(v_{t}\right), \lambda b_{t}\left(w_{t}\right)\right)+\{0\} \times(-\infty, 0]\right)\right)
$$

\section{References}

1. Beck, A., Ben-Tal, A.: Duality in robust optimization: Primal worst equals dual best. Operations Research Letters 37, 1-6 (2009)

2. Ben-Tal, A., El Ghaoui, L., Nemirovski, A.: Robust Optimization. Princeton Series in Applied Mathematics, Princeton (2009)

3. Ben-Tal, A., Boyd, S., Nemirovski, A.: Extending scope of robust optimization: comprehensive robust counterparts of uncertain problems. Math. Program. 107, 63-89 (2006)

4. Ben-Tal, A., Nemirovski, A.: Robust solutions of linear programming problems contaminated with uncertain data. Math. Program. 88, 411-424 (2000)

5. Ben-Tal, A.,Nemirovski, A.: Selected topics in robust convex optimization. Math. Program. 112B, 125-158 (2008)

6. Bertsimas, D., BrownD.: Constructing uncertainty sets for robust linear optimization. Oper. Res. 57, 1483-1495 (2009)

7. Birge, J.R., Louveaux, F.: Stochastic Programming. Springer, Berlin (1997)

8. Bogt, R.I., Wanka, G.: An alternative formulation for a new closed cone constraint qualification. Nonlinear Anal. 64, 1367-1381 (2006)

9. Burachik, R.S., Jeyakumar, V.: A new geometric condition for Fenchel's duality in infinite dimensional spaces. Math. Program. 104B, 229-233 (2005).

10. Dinh, N., Goberna, M.A., López, M.A.: From linear to convex systems: consistency, Farkas' lemma and applications. J. Convex Anal. 13, 279-290 (2006)

11. Dinh, N., Goberna, M.A.. López, M.A., Son, T.Q.: New Farkas-type constraint qualifications in convex infinite programming. ESAIM: Control Optim. Cal. Var. 13, 580-597 (2007)

12. El Ghaoui, L., Lebret, H.: Robust solutions to least-squares problems with uncertain data. SIAM J. Matrix Anal. Appl. 18, 1035-1064 (1997)

13. Goberna, M.A.: Linear semi-infinite optimization: recent advances. In: Jeyakumar, V., Rubinov, A.M. (eds.) Continuous Optimization, pp. 3-22. Springer, New York (2005) 
14. Goberna, M.A., Jeyakumar, V., López, M.A.: Necessary and sufficient constraint qualifications for solvability of systems of infinite convex inequalities. Nonlinear Anal. 68, 1184-1194 (2008).

15. Goberna, M.A., López, M.A.: Linear Semi-infinite Optimization. J. Wiley, Chichester (1998)

16. Jeroslow, R.G.: Uniform duality in semi-infinite convex optimization. Math. Program. 27, 144-154 (1983)

17. Jeyakumar, V.: Farkas Lemma: Generalizations. In: Floudas, C.A. Pardalos, P.M. (eds.) Encyclopedia of Optimization, Vol.2, pp. 87-91. Kluwer, Boston (2001)

18. Jeyakumar, V., Lee, G.M.: Complete characterizations of stable Farkas' lemma and cone-convex programming duality. Math. Program. 114, 335-347 (2008)

19. Jeyakumar, V., Li, G.: Characterizing robust set containments and solutions of uncertain linear programs without qualifications. Oper. Res. Lett. 38, 188$194(2010)$

20. Jeyakumar, V., Li, G.: Robust Farkas lemma for uncertain linear systems with applications. Positivity, in press. DOI: 10.1007/s11117-010-0078-4

21. Jeyakumar, V., Li, G.: Strong duality in robust convex programming: complete characterizations. SIAM J. Optim. 20, 3384-3407 (2010)

22. Jeyakumar, V., Wolkowicz, H.: Generalizations of Slater's constraint qualification for infinite convex programs. Math. Program. 57B, 85-101 (1992)

23. Li, G. , Ng, K.F.: On extension of Fenchel duality and its application. SIAM J. Optim. 19, 1489-1509 (2008).

24. Shapiro, A., Dentcheva, D., Ruszczynski, A.: Lectures on Stochastic Programming: Modeling and Theory. SIAM, Philadelphia (2009) 